\title{
HYBRID TECHNIQUE FOR IMPROVING UNDERWATER IMAGE
}

\section{A. Chrispin Jiji}

Assistant Professor, Department of Electronics and Communication Engineering, The Oxford College of Engineering, Bangalore, (India).

E-mail: chrispinjij@gmail.com

ORCID: https://orcid.org/0000-0001-5267-788x

\section{Nagaraj Ramrao}

Vice Chancellor, Kalasalingam University, Srivilliputtur, Tamilnadu, (India).

E-mail: Nagaraj.ramrao@gmail.com ORCID: https://orcid.org/0000-0003-2542-5999

\section{Gitación sugerida:}

Jiji, A. G., y Ramrao, N. (2021). Hybrid technique for improving underwater image. 3C Tecnología. Glosas de innovación aplicadas a la pyme, Edición Especial, (noviembre, 2021), 645-665. https://doi. org/10.17993/3ctecno.2021.specialissue8.645-665 


\section{ABSTRACT}

As light attenuate when disseminating in water, descriptions confined beneath water is generally corrupted towards varying degrees. An acquired image underneath water gets degraded due to optical properties of light in water. First, we describe optical behaviour of light in ocean due to which acquired images gets degraded. However, due to degradation of observed picture, conventional forms are not correct enough for faithful reconstruction. So, to improve the perception, we intend a Non-Locally Centralized Method (NLCM) for deblurring underwater descriptions. Later by considering characteristic of light propagation, we propose Gradient Guided Filter (GGF) method for improving the visibility of picture details. Finally, the image is well enhanced by Hybrid technique called Nonlocally centralized gradient guided filter (NLCM-GGF). Tentative outcome demonstrates that proposed technique produces improved results than several conventional techniques together in quality metrics and visual evaluation.

\section{KEYWORDS}

Underwater Image Processing, Deblurring, Edge preserving filters. 


\section{INTRODUCTION}

Light always acts a significant task in oceanic explorer. Light transmission in sea is the base of ocular studies for describing light transmission procedure. Underwater image analysis attracts an increased level of attention as well as support of ocean application such as undersea exploration; see life in undersea, ocean rescue in Lebart et al. (2003) and species identification in Strachan (1993). Thus, it has been a challenging task to restore as well enhance underwater images reasons the variation in ocular property underneath. The main effect of degradation processes causes turbid, decreases visibility along with color distortion owing to ocular property. In particular, for severe absorption of light, confined picture is under exposed. In the meantime, altered wavelength of light has diverse absorbing characteristic in Seibert (1963), attained picture has cruel color distortion. Larger wavelength determines poorer attenuation in transmission medium. Shorter wavelength travel further, because of these undersea descriptions subject to blue or green color in Torres-Méndez and Dudek (2005). The water turbidity in Huimin et al. (2015) and organic element suspended on medium yields a hard restoration problem, since overall technique becomes highly dependent on environmental conditions.

Numerous works encompass to undertake those problems. For past decades, an extensive study has performed to build up a variety of reconstruction methods in Bertero and Boccacci (1998), and Chan et al. (2005). Based on ill-posed nature, several methods are widely employed to improve the restored picture. For effective process, it is very important to model earlier facts of natural descriptions. The classic models, such as quadratic Tikhonov as well as TV model in Oliveira, Bioucas-Dias, and Figueiredo (2009) are effective to decrease noise artefacts however have a tendency to over-smooth the descriptions based on piecewise steady statement. As a substitute, in modern era sparsity model in Daubechies, Defrise, and De Mol (2004) and Dong et al. (2011) shows the potential outcome for different reconstruction issues in Mairal, Elad, and Sapiro (2008) and Mairal et al. (2009).

In this paper, we intend hybrid technique for improving underwater image. Our contributions are two-fold: (1) Non-Locally Centralized Method (NLCM) for deblurring the images; (2) Gradient Guided Filter (GGF) method for enhancing the images. Finally, the proposed NLCM-GGF method better improve its quality than conventional technique. 
Tentative result shows that projected technique performs better than many conventional techniques together in quality metrics and visual evaluation. In the rest of the paper, we present short outline of earlier art and describe optical properties of light under water in section 2. Section 3 describes proposed hybrid technique. Section 4 describes tentative result; finally, section 5 brings to a close note.

\section{MATERIALS AND METHODS}

A variety of techniques has projected for getting better ocular excellence of degraded undersea descriptions, roughly classified into deblurring and enhancement process. Deblurring undersea picture is an ill-posed problem. As an essential issue in undersea description, reconstructions have widely considered in earlier years in Banham and Katsag (1997) and Bioucas-Dias and Figueiredo (2007). The ill-posed nature of IR is normally not exceptional. Past facts of typical descriptions employed to regularize such issues in reconstruction. The main model is total variation (TV) which lack flexibility for characterizing local picture structures but often generates over-smoothed results. To well keep up the picture boundaries, several methods have developed for improving TV model in Lysaker and Tai (2006), Beck and Teboulle (2009) and Chantas et al. (2010). The autoregressive (AR) modelling in Wu, Zhang, and Wang (2009) closely computes a primary representation which gives improved results than TV model to restore boundary formation, but have a tendency to generate ghost artefact.

In Buades, Coll, and Morel (2005) training-based adaptive scheme learns a better-quality learning pictures, to increase its accuracy. In modern era non-local (NL) in Kindermann, Osher, and Jones (2005) and Zhang et al. (2010) process has led a hopeful effect in several reconstruction schemes. The plan of NL process is straightforward: patch that contain related pattern be spatially distant and so we bring together in the image. In Dong et al. (2011) and Jiji and Vivek (2017), the edges are sharper than all the other process but there shows some ringing noise around edges and with different patches for reconstructing the images in Jiji and Ramrao (2017). With this aim, centralized NL method exploits NL redundancy to lower SCN noise. 
The NCSR method in Dong et al. (2013), use NL self-similarity for gaining fine approximation of sparse code coefficients, later integrate attained picture to those approximation. It is also characterized by training online sub-dictionaries by choosing most excellent online subdictionary for every patch. It employs Iterative Shrinkage-Threshold (IST) for solving l-norm trouble produced by model. Although the method achieved good, but never considers the statistical picture formation, therefore undergo artefact near boundaries.

Enhancement method does not rely on any picture formation, and enhances imagery by modifying scene pixel values. In Farbman et al. (2008) fine points in true description will smooth while keep boundaries to subtract smooth picture from true description for generating detailed picture. The mixture of range and domain filter in Tomasi and Manduchi (1998) keeps boundaries sharper, but experience gradient problems next to few boundaries. To avoid gradient reversal artifacts in He, Sun, and Tang (2013), picture elements in a window consider the structure of guidance image, but fail to signify close to a few boundaries. The boundary responsive factor in Li et al. (2015) lowers halo artifact which makes the edges better, but they cannot keep up edges well in some cases. The factors in Kou et al. (2015) signify the descriptions more correctly next to boundaries and keep up good boundaries. The work in Jiji and Ramrao (2019) is the extensive version primarily existed to improve the undersea imagery.

Here, we describe a hybrid technique for improving undersea descriptions which built upon the idea of NLGM exploit regulation limit determines the geometrical formation of image. A new method is particularly functional on boundaries of restored representation, so known as NLCM based Gradient guided filter (NLCM-GGF). Experimental results proved that the projected scheme gives much enhancement in both quality metrics and visual excellence than conventional technique.

\subsection{OPTICAL PROPERTIES IN WATER}

This section describes the behaviour of beam in undersea. Beam propagates in water medium is same as in air. Absorption denotes power reduction and scattering refers to deflection of propagation path. In underwater environment, beam also undergoes diffraction as well as refraction due to its wavelength and refractive index of water. Lambert-Beer empirical law states that the ocular property decay underneath material via exponential dependence: 


$$
E_{r}=E_{o} e^{-c d}
$$

where $c$ denotes overall attenuation coefficient, $d$ denotes the distance from an object. This model further decomposes in a form that openly expressed as

$$
E_{r}=E_{o} e^{-a d} e^{-b d}
$$

In order to deal with these effects another property volume scattering $B(\theta)$ as:

$$
f=\frac{1}{2 \pi} \int_{0}^{\pi} B(\theta) \sin (\theta) d \theta
$$

The angle $\theta$ be integrated to get total scattering $f$. It theoretically considers all contributions coming from all directions. Modelling the backward scatter is more complicated than the forward one because it requires explicit volume scattering function. Four quantities $a, b$, $c, B(\theta)$ represents an ocular property of underneath medium. This resulting form used to predict ocular behaviour of light underneath.

For scattering, absorption as well as other optical properties always strictly related to specific medium composition. This fact justifies variability that we meet in dealing with them. Therefore, to concern picture arrangement process itself, direct, back-scattered as well as forward-scattered beam forms three additive total irradiance mechanisms, which mathematically expressed as:

$$
E_{T}=E_{D}+E_{B}+E_{F}
$$

Where light received by camera consists of three components: (i) object reflect beam with scattering, (ii) object reflect beam without scattering, (iii) back scatter part. For further details in Jaffe (1990) crucial quantities $E_{D}, E_{B}$ and $E_{F}$ as well analytical formulas that discussed in deep with definition of an expression for each part of whole irradiance. 


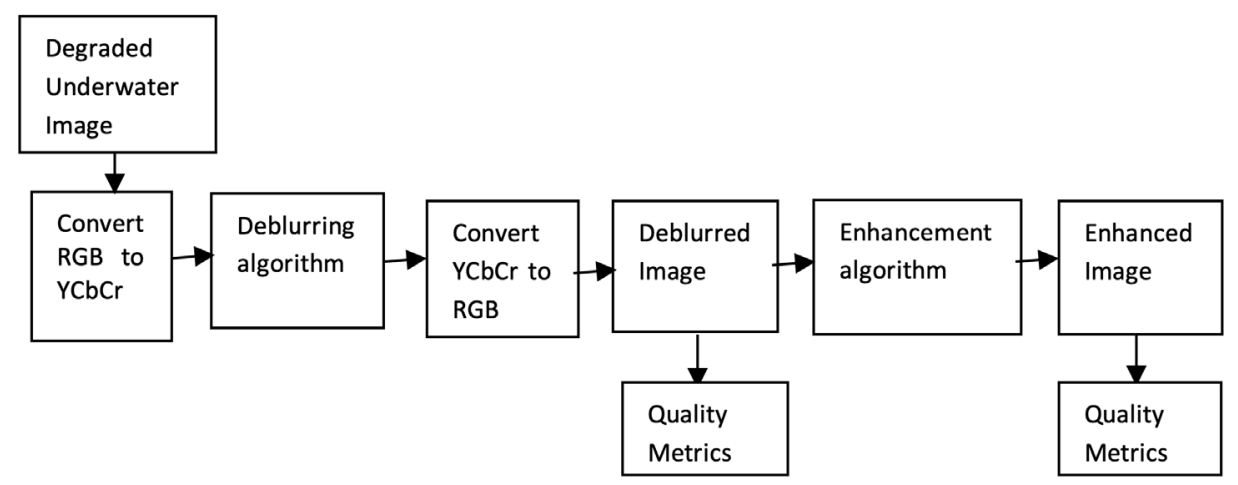

Figure 1. Block diagram of Proposed Method.

Source: own elaboration.

\section{PROPOSED METHOD}

The optical behaviour of beam in water will degrade the obtained pictures taken from underwater. In order to solve these issues, we intend a Hybrid technique that is able to deblur and enhance the underwater images depicted in Figure 1. Our hybrid approach consists of two main steps. In the first step, the Non-locally centralized method used for deblurring the scene. It mainly improves sparse restoration and suppresses the sparse coding noise. The main feature is to train online sub-dictionaries and choosing online sub-dictionary to each patch, using IST method for solving 11-minimization trouble created using such models. But it generates some ringing artefacts around the restored boundaries. In the second step, we introduce a Gradient guided filter (GGF) to improve edge sharpness. Finally, the output is well enhanced through Non-Locally Centralized Method via Gradient Guided Filter (NLGM-GGF). Experimental results proved that the projected scheme gives much enhancement in both quality metrics and visual excellence than conventional technique.

\subsection{DEBLURRING ALGORITHM}

The deblurring algorithm as depicted in Figure 2, blurred \& noisy description (b) attained with blurring an ideal picture $(r)$ with point spread function $(H)$, then imposing noise $(n)$, corresponding mathematical formula expressed as follows:

$$
b=H r+n
$$


In fact, some unknown quantities higher than known quantities; this problem becomes an ill-posed problem, which needs some other priori information.

To recover the reconstruction, first $b$ is sparsely coded to solve minimization problem as:

$$
\alpha_{y}=\arg \min \left\{\|b-H \phi \alpha\|_{2}^{2}+\lambda\|\alpha\|_{1}\right\}
$$

Conversely, reconstructing $\alpha_{r}$ from $b$ is the very difficult task. For faithful reconstruction, we used in Dong et al. (2013) to reduce sparse coded image. The sparse coding noise stage represents

$$
n_{\alpha}=\alpha_{b}-\alpha_{r}
$$

By reducing $n_{\alpha}$ we can get better output. To suppress $n_{\alpha}$, improve $\alpha_{b}$, we propose the following model:

$$
\alpha_{b}=\arg \min \left\{\|r-\phi \alpha\|_{2}^{2}+\lambda\left\|\alpha_{i}\right\|_{1}+\gamma\left\|\alpha_{i}-\beta_{i}\right\|_{p}\right\}
$$

where $\beta_{i}$ signify fine evaluation of $\alpha_{i}, \boldsymbol{\gamma}$ signify regularize constraint and $p$ will be 1 or 2 .

To select dictionary, we cluster the patches by $K$-means clustering, then uses PCA in each cluster to find the sub dictionary. To code each patch, we enforce that particular patch by keeping sub-dictionaries as zero.

Hence sparse coding model as:

$$
\alpha_{b}=\arg \min \left\{\|r-\phi \alpha\|_{2}^{2}+\gamma \sum_{i}\left\|\alpha_{i}-\beta_{i}\right\|_{p}\right\}
$$

Where $\beta_{i}$ represents mass, average related by NL like patches

$$
\beta_{i}=\sum_{t \in \Omega_{i}} m_{i, t} \alpha_{i, t}
$$

where $m_{i, t}$ denotes weight. Related to NL means, we set weights as:

$$
m_{i, t}=\frac{1}{w} \exp \left(-\left\|\hat{r_{i}}-\hat{r_{i, t}}\right\|_{2}^{2} / h\right)
$$


where $\hat{r_{i}}$ and $\hat{r_{i, t}}$ denotes estimation of patch $r_{i}$ and $r_{i, t} h$ represents predestined scalar and w denotes normalization part.

For each iteration, sparse vector represents:

$$
\alpha_{b}^{(l)}=\arg \min \left\{\|r-\phi \alpha\|_{2}^{2}+\gamma \sum_{i}\left\|\alpha_{i}-\beta_{i}^{(l)}\right\|_{p}\right\}
$$

For IST process, learning patches updated by present description of reconstruction and update PCA bases as well as by repeating neighbourhood choice with reorganized learning information. For every iteration by updating learning set with PCA bases, current test patch updated by $y=\left\{y_{j}\right\}=\left\{r_{j}\right\}_{j=1}^{M}$. The restored representation is then updated as $\stackrel{\wedge(l)}{r}=\phi \circ \alpha_{b}^{(l)}$

\subsection{GRADIENT GUIDED FILTER (GGF)}

The deblurred output better reconstruct the image but it generates a few ring artefacts around the restored boundaries. With this aim, a Gradient guided filter (GGF) in Kou et al. (2015) is to improve edge sharpness. The filtered as well as guidance pictures are same for detailed output. The projected method gives an edge-preserved smooth picture. The difference among input with output gives detail layer, which is mainly for strengthening the output. In an edge, $a_{d^{\prime}}$ denotes

$$
a_{d^{\prime}}=\frac{\sigma_{g, \xi_{1}}^{2}\left(d^{\prime}\right)+\frac{\lambda}{\hat{\Gamma}\left(d^{\prime}\right)}}{\sigma_{g, \xi_{1}}^{2}\left(d^{\prime}\right)+\frac{\lambda}{\hat{\Gamma}\left(d^{\prime}\right)}}
$$

The rate of $a_{d^{\prime}}$ is nearer to 1 if pixel $a_{d^{\prime}}$ is in boundary, such that sharp boundaries are good in projected method than conventional technique.

In flat area, $a_{d^{\prime}}$ is usually 0 and $\Gamma^{\star}\left(d^{\prime}\right)$ is smaller than 1 denoted as:

$$
a_{d^{\prime}}=\frac{\sigma_{g, \xi_{1}}^{2}\left(d^{\prime}\right)}{\sigma_{g, \xi_{1}}^{2}\left(d^{\prime}\right)+\frac{\lambda}{\hat{\Gamma}\left(d^{\prime}\right)}}
$$




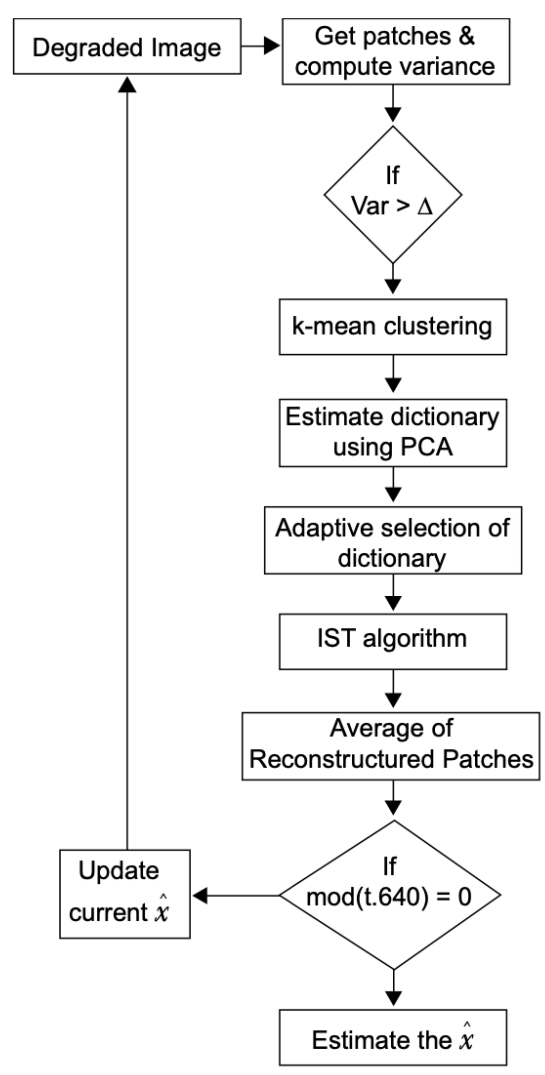

Figure 2. Flowchart of Deblurring algorithm.

Source: own elaboration.

In an edge, larger $\lambda$ chooses for projected method than existing because choice will not affect edges. This means that projected method smooth flat area better than existing technique.

For these two cases the weighing function $\Gamma^{(}\left(d^{\prime}\right)$ denoted as:

$$
\hat{\Gamma}\left(d^{\prime}\right)=\frac{1}{N} \sum_{d=1}^{N} \frac{\sigma_{g, \xi_{1}}^{2}\left(d^{\prime}\right)+\varepsilon}{\sigma_{g, \xi_{1}}^{2}(d)+\varepsilon}
$$

Where $\xi_{1}$ signify filter window dimension. The projected method is sharper by way of increasing of $\lambda$. Though, it has fewer artefacts even by larger $\lambda$. So, we used larger $\lambda$ in projected method exclusive of halo artefacts. 


\subsection{ENHANCEMENT METHOD}

After the process of NLGM and GGF, we joined both to improved eminence of representation. For better reconstruction we employed NLCM method which generate sharper boundaries and restore best descriptions, but it produces some ringing artefacts around the reconstructed edges. To avoid these effects, deblurred output takes advantage of GGF for edge-preservation. The projected NLCM-GGF offer enhanced outcome than existing techniques for both evaluation metrics and visual perception.

\section{RESULTS}

Our projected scheme compared by various conventional reconstruction methods: ASDSARNL in Dong et al. (2011), NGSR in Dong et al. (2013) and DGFGGF in Jiji and Ramrao (2019). Our method also combines with BF, GF, WGF and GGF. Consequently, it is obligatory for comparing different edge filters to better keep edges. Here we carried out performance of various techniques, image evaluations.

\subsection{Subjective Performance Comparison}

Various methods of reconstruction include: ASDSARNL in Dong et al. (2011), NGSM in Dong et al. (2013), DCFGGF in Jiji and Ramrao (2019), NLCM-BF, NLCM-GF, NLCMWF and NLGM-GGF.

To make sure the fairness of each assessment system, all test underwater images are pre-processed at size $256 \times 256$ pixels and processed by evaluated schemes with default parameters. The results in Dong et al. (2011) generate better visual excellence and evaluation metrics, though lesser patch dimension produces few artefacts in smooth areas. The results in Dong et al. (2013) much outperform Dong et al. (2011), produce sharper boundaries and further restore its quality. The projected smoothing layer in Figure 3 yields improved results than conventional schemes. Similarly, detail enhancement in Figure 4 is also much clearer and better than conventional schemes but there exhibit fewer edges in WGF than others. In Figure 5 we present hybrid result of existing and projected means. 


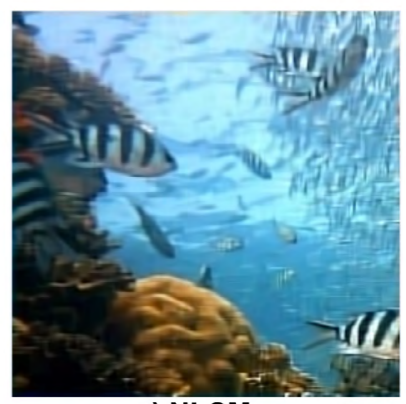

a) NLCM

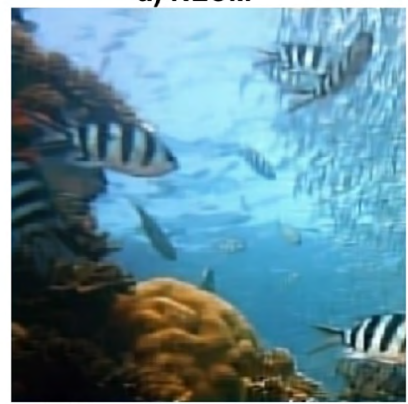

d) NLCM-WGF Smoothing

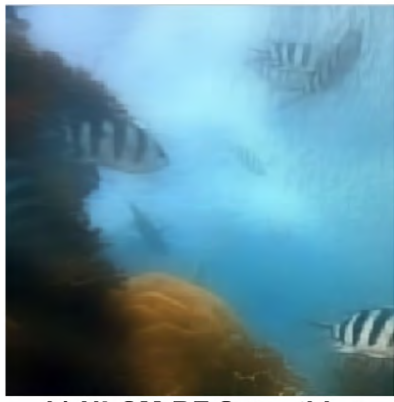

b) NLCM-BF Smoothing

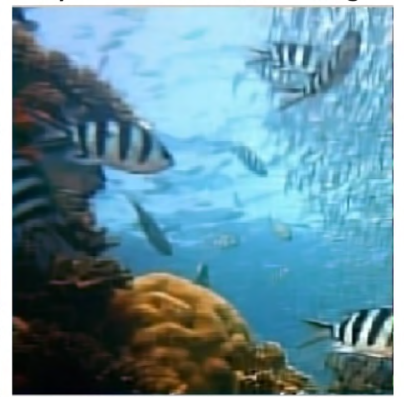

e) NLCM-GGF Smoothing

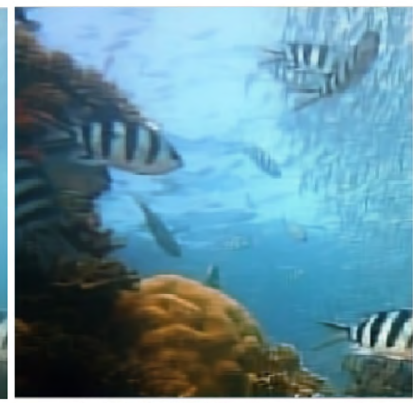

c) NLCM-GF Smoothing

Figure 3. Edge smoothing results of Existing and Proposed Method. Source: own elaboration.

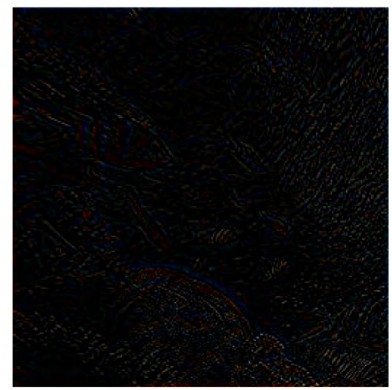

a) NLCM Details

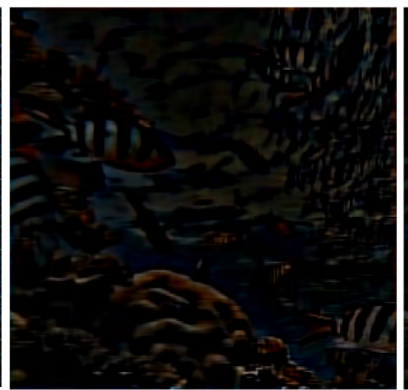

b) NLCM-BF Details

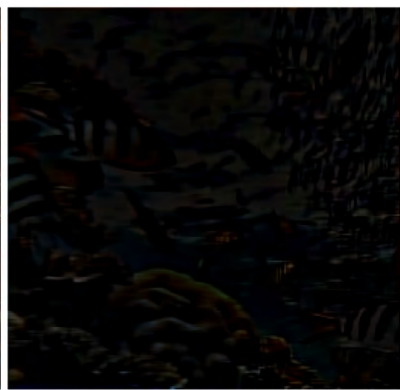

c) NLCM-GF Details

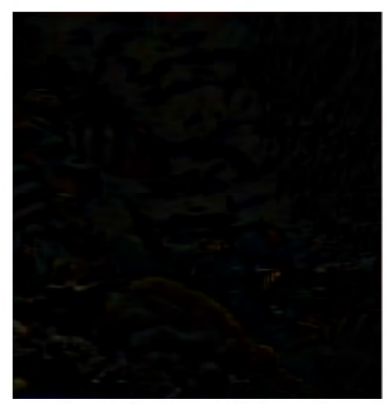

d) NLCM-WGF Details

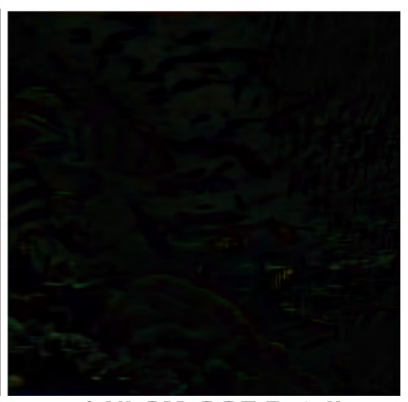

e) NLCM-GGF Details

Figure 4. Detail Enhancement results of Existing and Proposed Method. Source: own elaboration. 


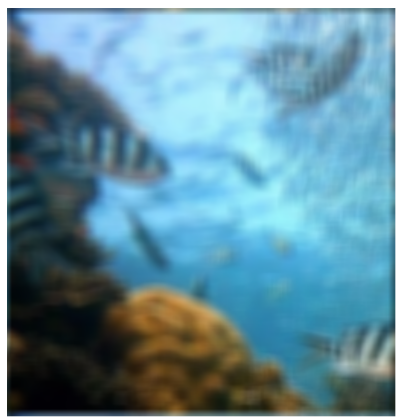

a) Degraded Image

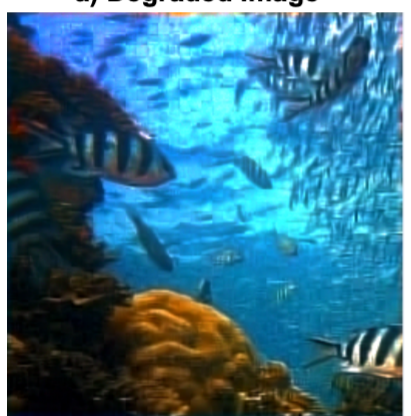

d) (Jiji \& Ramrao, 2019)

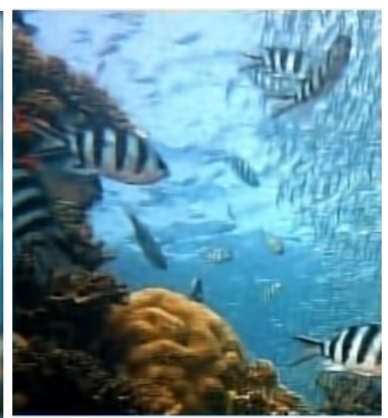

b) (Dong et al., 2011)

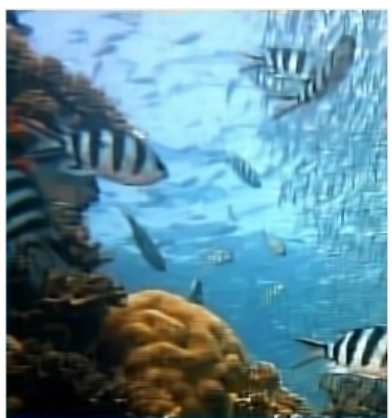

c) (Dong et al., 2013)

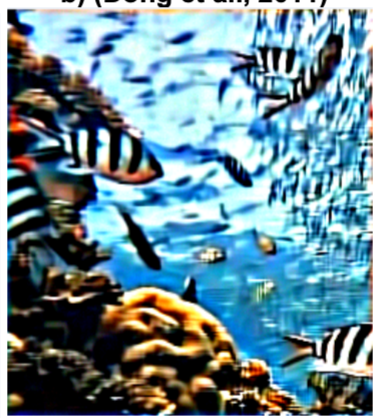

e) NLCM-BF

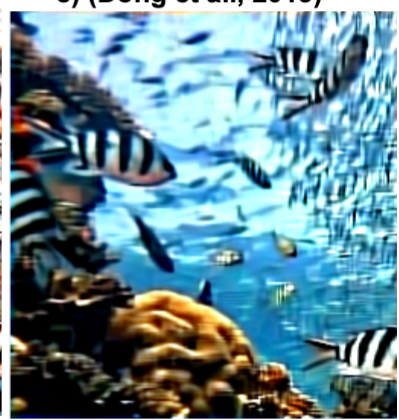

f) NLCM-GF

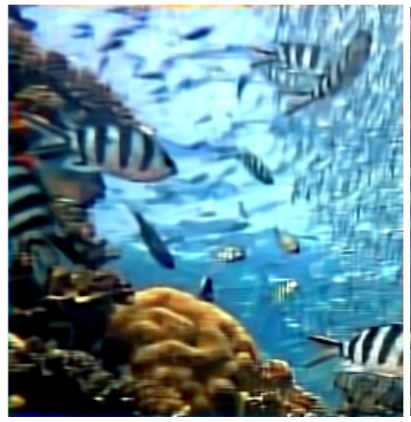

g) NLCM-WGF

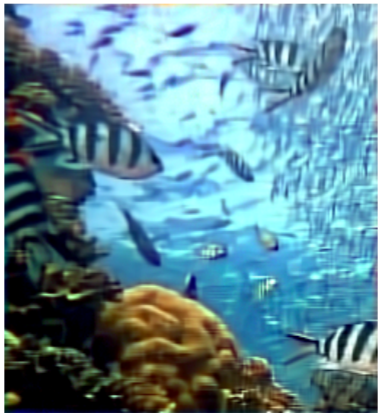

h) NLCM-GGF

Figure 5. Enhancing performance evaluation of Underwater Imagery. Source: own elaboration.

We observed that proposed process results much improved and enhanced details than conventional process.

\subsection{OBJECTIVE PERFORMANCE COMPARISON}

Image quality usually affected through imaging equipment, instrument noise, imaging conditions, image processing and other factors. Image Quality Assessment (IQA) is often 
separated into subjective qualitative assessment. Gray mean rate of picture reflects integral intensity and expressed as:

$$
\text { Mean }=\frac{1}{R C} \sum_{r=1}^{R} \sum_{c=1}^{C} O(r, c)
$$

Standard deviation reflects high frequency part that relates picture contrast. Higher values give better contrast.

$$
S D=\frac{1}{R C} \sum_{r=1}^{R} \sum_{c=1}^{C}\{O(r, c), \text { Mean }\}^{2}
$$

Mean gradient reflects speed of changes in minor details of picture; it can represent description of grain transform and quantity of clearness well.

$$
A G=\frac{1}{R C} \sum_{r=1}^{R} \sum_{c=1}^{C} \sqrt{\frac{[O(r, c)-O(r+1, c)]^{2}+[O(r, c)-O(r, c)+1]^{2}}{2}}
$$

Entropy interprets as average uncertainty of data. When applied to images, it represents abundance data observed in picture. Higher entropy gives more uniform contrast

$$
\text { Entropy }=-\sum_{r=1}^{L} P\left(O_{r}\right) \log _{2} P\left(O_{r}\right)
$$

Mean Square averages squared intensity differences of among distorted and reference representation as

$$
R M S E=\sqrt{\frac{1}{R C} \sum_{r=1}^{R} \sum_{c=1}^{C}\left[O(r, c)-O^{\prime}(r, c)\right]}
$$

where $R, C$ denotes row and column, $O(r, c)$ remains original with $O^{\prime}(r, c)$ denotes deblurred picture. Peak SNR (PSNR) signify a key meant for signal alteration.

$$
\text { Peak SNR }=10 \log \frac{O_{\max }^{2}}{M S E}
$$

Where $O_{\max }$ represents maximum gray rate. Higher PSNR value represents lesser distortion. Generally, ocular view in Wang and Yuan (2017) and Wang et al. (2004) particularly adapted to extract picture information, thus process image excellence by three mechanism specifically; luminance $l(I, O)$, contrast $C(I, O)$, structure comparison $S(I, O)$. Thus, similarity computation represents 


$$
S(r, c)=F(l(I, O), C(I, O), S(I, O))
$$

The similarity of two images has a rate among $[0,1]$. When it is close to 1 , two descriptions are more similar.

In Yang and Sowmya (2015) underwater color image quality evaluation (UCIQE) represents contrast, chroma and saturation expressed as:

$$
U C I Q E=c_{1} \sigma_{c}+c_{2} \operatorname{con}_{l}+c_{3} \mu_{c}
$$

Where $\sigma_{c}, \operatorname{con}_{l}$ and $\mu_{c}$ represent standard deviation, contrast and mean, $c_{1}, c_{2}, c_{3}$ represent these three weights. Higher UCIQE metrics have improved results than conventional schemes. Similar to UCIQE, undersea image quality measure (UIQM) in Panetta, Gao, and Agaian (2016) constructed linear combination of UI colorfulness metric (UICM), UI sharpness metric (UISM) and UI contrast metric (UIConM). Thus, larger UCIQE and UIQM, improved undersea color image quality will be.

$$
U I Q M=\alpha U I C M+\beta U I S M+\gamma U I C o n M
$$

Where $\alpha, \beta, \gamma$ signifies weight coefficients to organize each measure as well as balance their rates. Higher UICM value indicates improved color of undersea descriptions. Table 1

\begin{tabular}{|c|c|c|c|c|c|c|c|}
\hline \multirow[b]{2}{*}{$\begin{array}{l}\text { Quality } \\
\text { metrics }\end{array}$} & \multicolumn{2}{|c|}{ DEBLURRED IMAGE } & \multicolumn{5}{|c|}{ ENHANCED IMAGE } \\
\hline & $\begin{array}{l}\text { (Dong et } \\
\text { al., 2011) }\end{array}$ & $\begin{array}{l}\text { (Dong et } \\
\text { al., 2013) }\end{array}$ & $\begin{array}{c}(\mathrm{Jiji} \& \\
\text { Ramrao, } \\
\text { 2019) }\end{array}$ & NLCM-BF & NLCM-GF & NLCM-WGF & NLCM-GGF \\
\hline Mean & 127.34 & 127.36 & 96.639 & 126.81 & 126.73 & 127.22 & 127.29 \\
\hline SD & 71.236 & 71.153 & 67.783 & 94.869 & 86.831 & 77.084 & 74.339 \\
\hline AG & 9.226 & 8.065 & 10.194 & 23.761 & 18.507 & 12.988 & 11.681 \\
\hline Entropy & 7.933 & 7.941 & 7.541 & 6.351 & 6.984 & 7.536 & 7.619 \\
\hline PSNR & 24.10 & 26.231 & 65.843 & 66.916 & 70.956 & 72.255 & 72.417 \\
\hline RMSE & 13.717 & 0.752 & 0.139 & 0.115 & 0.072 & 0.062 & 0.061 \\
\hline SSIM & 0.619 & 12.443 & 0.9977 & 0.9990 & 0.9997 & 0.9998 & 0.9998 \\
\hline UICM & -45.558 & -45.555 & -62.107 & -33.899 & -39.299 & -43.522 & -44.177 \\
\hline
\end{tabular}
shows assessment metrics of conventional and projected technique.

Table 1. Comparison of quality metric with existing and proposed methods. 


\begin{tabular}{|c|c|c|c|c|c|c|c|}
\hline UIConM & 0.736 & 0.728 & 0.785 & 0.305 & 0.583 & 0.701 & 0.681 \\
\hline UISM & 6.906 & 7.007 & 7.105 & 7.827 & 7.289 & 7.057 & 7.073 \\
\hline UIQM & 3.389 & 3.388 & 3.155 & 2.449 & 3.131 & 3.364 & 3.278 \\
\hline UCIQE & 32.073 & 32.112 & 35.233 & 35.597 & 33.729 & 32.548 & 32.423 \\
\hline
\end{tabular}

Source: own elaboration.

\section{CONCLUSIONS}

An acquired image underneath water gets degraded due to optical properties of light in water. However, due to degradation of observed picture, conventional forms are not correct enough for faithful reconstruction. In this paper, we proposed a hybrid technique for improving undersea descriptions. First, we used a Non locally centralized (NLCM) method for deblurring underwater descriptions, later gradient guided filter algorithm to improve the visibility of picture details and finally a Hybrid technique called non-locally centralized gradient guided filter (NLGM-GGF) gives improved results. Experimental results of proposed technique perform better than many conventional techniques together in quality metrics and visual evaluation. Nevertheless, when the illumination is very uneven, enhancement limits the local dark region, and it requires further research.

\section{ACKNOWLEDGEMENT}

We gratefully thank the Visvesvaraya Technological University, Jnana Sangama, Belagavi for their extended support to this Research work.

\section{REFERENCES}

Banham, M. R., \& Katsag, A. K. (1997). Digital image restoration. IEEE Signal Processing Magazine, 14(2), 24-41. https://doi.org/10.1109/79.581363

Beck, A., \& Teboulle, M. (2009). Fast Gradient-Based Algorithms for Constrained Total Variation Image Denoising and Deblurring Problems. IEEE Transactions on Image Processing, 18(11), 2419-2434. https://doi.org/10.1109/TIP.2009.2028250

Bertero, M., \& Boccacci, P. (1998). Introduction to Inverse Problems Imaging. GRC Press. 
Bioucas-Dias, J. M., \& Figueiredo, M. A. T. (2007). A New TwIST: Two-Step Iterative Shrinkage/Thresholding Algorithms for Image Restoration. IEEE Transactions on Image Processing, 16(12), 2992-3004. https://doi.org/10.1109/TIP.2007.909319

Buades, A., Coll, B., \& Morel, J.-M. (2005). A review of image denoising algorithms, with a new one. Multiscale Modeling and Simulation: A SIAM Interdisciplinary fournal, Society for Industrial and Applied Mathematics, 4(2), 490-530. ffhal-00271141f. https:// hal.archives-ouvertes.fr/hal-00271141/document

Chan, T., Esedoglu, S., Park, F., \& Yip A. (2005). Recent developments in total variation image restoration. http://vision.mas.ecp.fr/paragios-chen-faugeras/sample.pdf

Chantas, G., Galatsanos, N. P., Molina, R., \& Katsaggelos, A. K. (2010). Variational Bayesian Image Restoration With a Product of Spatially Weighted Total Variation Image Priors. IEEE Transactions on Image Processing, 19(2), 351-362. https://doi. org/10.1109/TIP.2009.2033398

Daubechies, I., Defrise, M., \& De Mol, G. (2004). An iterative thresholding algorithm for linear inverse problems with a sparsity constraint. Communications on Pure and Applied Mathematics, 57(11), 1413-1457. https://doi.org/10.1002/cpa.20042

Dong, W., Zhang, L., \& Shi, S. (2011). Centralized sparse representation for image restoration. In International Conference on Computer Vision, 1259-1266. https://doi. org/10.1109/ICGV.2011.6126377

Dong, W., Zhang, L., Shi, G., \& Li, X. (2013). Nonlocally Centralized Sparse Representation for Image Restoration. IEEE Transactions on Image Processing, 22(4), 1620-1630. https://doi.org/10.1109/TIP.2012.2235847

Dong, W., Zhang, L., Shi, G., \& Wu, X. (2011). Image Deblurring and Super-Resolution by Adaptive Sparse Domain Selection and Adaptive Regularization. IEEE Transactions on Image Processing, 20(7), 1838-1857. https://doi.org/10.1 109/TIP.2011.2108306

Farbman, Z., Fattal, R., Lischinski, D., \& Szeliski, R. (2008). Edge-preserving decompositions for multi-scale tone and detail manipulation. ACM Transactions on Graphics, 27(3), 1-10. https://doi.org/10.1145/1360612.1360666 
He, K., Sun, J., \& Tang, X. (2013). Guided image filtering. http://kaiminghe.com/ publications/eccvloguidedfilter.pdf

Huimin, L., Yujie, L., Lifeng, Z., \& Seiichi, S. (2015). Contrast enhancement for images in turbid water. Fournal of the Optical Society of America A, 32(5), 886-893. https: / / www.osapublishing.org / viewmedia.cfm? $r=1 \&$ rwjcode=josaa\&uri=josaa32-5-886\&html=true

Jaffe, J. S. (1990). Computer modeling and the design of optimal underwater imaging systems. IEEE Fournal of Oceanic Engineering, 15(2), 101-111. https://doi. org/10.1109/48.50695

Jiji, A. G., \& Ramrao, N. (2017). Deblurring Underwater image degradations based on adaptive regularization. ICICI.

Jiji, A. C., \& Ramrao, N. (2019). A Novel Imaging System for Underwater Haze Enhancement. International Fournal of Information Technology, 12, 85-90. https://link. springer.com/article/10.1007/s41870-019-00312-y

Jiji, A. G., \& Vivek, M. (2017). Underwater turbidity removal through ill-posed optimization with sparse modelling. In 2017 IEEE International Conference on Power, Control, Signals and Instrumentation Engineering (ICPCSI), pp. 1865-1869. https://doi. org/10.1109/ICPCSI.2017.8392039

Kindermann, S., Osher, S., \& Jones, P. W. (2005). Deblurring and denoising of images by nonlocal functional. Multiscale Modeling $\&$ Simulation, 4(4), 1091-1115. https://doi. $\mathrm{org} / 10.1137 / 050622249$

Kou, F., Ghen, W., Wen, G., \& Li, Z. (2015). Gradient Domain Guided Image Filtering. IEEE Transactions on Image Processing, 24(11), 4528-4539. https://doi.org/10.1109/ TIP.2015.2468183

Lebart, K., Smith, G., Trucco, E., \& Lane, D. (2003). Automatic indexing of underwater survey video: algorithm and benchmarking method. IEEE fournal of Oceanic Engineering, 28(4), 673-686. https://doi.org/10.1109/JOE.2003.819314 
Li, Z., Zheng, J., Zhu, Z., Yao, W., \& Wu, S. (2015). Weighted Guided Image Filtering. IEEE Transactions on Image Processing, 24(1), 120-129. https://doi.org/10.1109/ TIP.2014.2371234

Lysaker, M., \& Tai, X. C. (2006). Iterative Image Restoration Combining Total Variation Minimization and a Second-Order Functional. International fournal of Computer Vision, 66, 5-18. https://doi.org/10.1007/s1 1263-005-3219-7

Mairal, J., Bach, F., Ponce, J., Sapiro, G., \& Zisserman, A. (2009). Non-local sparse models for image restoration. In 2009 IEEE 12th International Conference on Computer Vision, pp. 2272-2279. https://doi.org/10.1109/ICGV.2009.5459452

Mairal, J., Elad, M., \& Sapiro, G. (2008). Sparse Representation for Color Image Restoration. IEEE Transactions on Image Processing, 17(1), 53-69. https://doi. org/10.1109/TIP.2007.911828

Oliveira, P. J., Bioucas-Dias, J. M., \& Figueiredo, M. A. T. (2009). Adaptive total variation image deblurring: A majorization-minimization approach. Signal Processing, 89(9), 1683-1693. https://doi.org/10.1016/j.sigpro.2009.03.018

Panetta, K., Gao, G., \& Agaian, S. (2016). Human-Visual-System-Inspired Underwater Image Quality Measures. IEEE Journal of Oceanic Engineering, 41(3), 541-551. https:/ / doi.org/10.1109/JOE.2015.2469915

Seibert, Q. (1963). Light in the sea. Fournal of the Optical Society of America, 53(2), 214-233. https://doi.org/10.1364/JOSA.53.000214

Strachan, N. (1993). Recognition of fish species by color and shape. Image and Vision Computing, 11(1), 2-10. https://doi.org/10.1016/0262-8856(93)90027-E

Tomasi, G., \& Manduchi, R. (1998). Bilateral filtering for gray and color images. Proceedings of the 1998 IEEE International Conference on Computer Vision. https:/ / users.soe. ucsc.edu/ manduchi/Papers/ICGV98.pdf

Torres-Méndez, L. A., \& Dudek, G. (2005) Color Correction of Underwater Images for Aquatic Robot Inspection. In: Rangarajan, A., Vemuri, B., \& Yuille, A.L. (eds.), 
Energy Minimization Methods in Computer Vision and Pattern Recognition. EMMCVPR 2005. Lecture Notes in Computer Science, vol. 3757. Springer, Berlin, Heidelberg. https://doi.org/10.1007/11585978_5

Wang, W., \& Yuan, X. (2017). Recent advances in image dehazing. IEEE/CAA fournal of Automatica Sinica, 4(3), 410-436. https://doi.org/10.1109/JAS.2017.7510532

Wang, Z., Bovik, A. C., Sheikh, H. R., \& Simoncelli, E. P. (2004). Image quality assessment: from error visibility to structural similarity. IEEE Transactions on Image Processing, 13(4), 600-612. https://doi.org/10.1109/TIP.2003.819861

Wu, X., Zhang, X., \& Wang,J. (2009). Model-Guided Adaptive Recovery of Compressive Sensing. In 2009 Data Compression Conference, pp. 123-132. https://doi.org/10.1109/ DCG.2009.69

Yang, M., \& Sowmya, A. (2015). An Underwater Color Image Quality Evaluation Metric. IEEE Transactions on Image Processing, 24(12), 6062-6071. https://doi.org/10.1109/ TIP.2015.2491020

Zhang, X., Burger, M., Bresson, X., \& Osher, S. (2010). Bregmanized nonlocal regularization for deconvolution and sparse reconstruction. SIAM fournal on Imaging Sciences, 3(3), 253-276. https://epubs.siam.org/doi/10.1137/090746379 
